MULTIPHASE IMAGE SEGMENTATION

VIA MODICA-MORTOLA PHASE TRANSITION

By

Yoon Mo Jung

Sung Ha Kang

and

Jianhong Shen

IMA Preprint Series \# 2124

(June 2006)

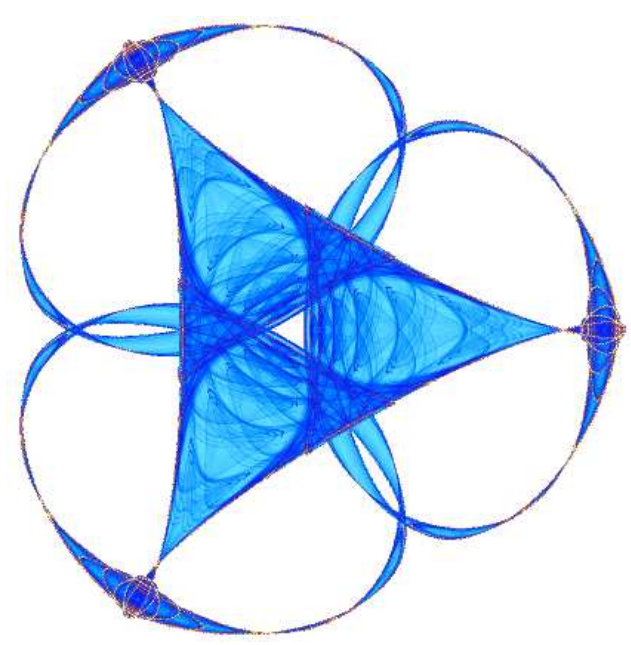

INSTITUTE FOR MATHEMATICS AND ITS APPLICATIONS

UNIVERSITY OF MINNESOTA

400 Lind Hall

207 Church Street S.E.

Minneapolis, Minnesota 55455-0436

Phone: 612/624-6066 Fax: 612/626-7370

URL: http://www.ima.umn.edu 


\title{
Multiphase Image Segmentation via Modica-Mortola Phase transition
}

\author{
Yoon Mo Jung, Sung Ha Kang, and Jianhong Shen *
}

\begin{abstract}
We propose a novel multiphase segmentation model built upon the celebrated phase transition model of Modica and Mortola in material sciences and a properly synchronized fitting term that complements it. The proposed sine-sinc model outputs a single multiphase distribution from which each individual segment or phase can be easily extracted. Theoretical analysis is developed for the $\Gamma$-convergence behavior of the proposed model and the existence of its minimizers. Since the model is not quadratic nor convex, for computation we adopted the convex-concave procedure (CCCP) that has been developed in the literatures of both computational nonlinear PDEs and neural computation. Numerical details and experiments on both synthetic and natural images are presented.
\end{abstract}

\section{Introduction}

The literature on segmentation has been the most wealthy and inspiring. From Geman and Geman's mixture random-field models [23] to Mumford and Shah's piecewise smooth variational image models [36], segmentation has been extensively studied by several major stochastic and deterministic machineries of modeling, analysis, and computation. New segmentation models incorporating more complexities or flexibilities have been further proposed by a number of authors in recent years, e.g., the data-driven Monte-Carlo Markov chain model (DDMCMC) of Tu and Zhu [51], the graph-cutting and spectral method of Shi and Malik [47], and the variational texture segmentation models by Sandberg, et al. [42], and Shen [45] (based on the texture models of Meyer [32] and Osher et al. [40]), just to name a few.

In this paper, we focus on the variational-PDE approach that is closely connected to the Mumford-Shah type of models. Computationally, such models have been implemented in various approaches: the finitedifference or finite-element methods, e.g., by Chambolle [5, 8, 9] and Morel and Solimini [35], as well as the influential level-set approach by Chan and Vese $[15,13]$ (based on the level-set technology of Osher and Sethian $[38,39,43])$. In the level-set approach, in particular, several multiphase computational models have been recently designed by Chan and Vese [16], Chung and Vese [17], as well as Lie et al. [28, 49]. We emphasize that the current work is more or less related to those ideas explored in [17] and [28], but it is carried out in a completely different framework.

An alternative approach to modeling and computing segmentation is via the theory of $\Gamma$-convergence elliptic approximations, as first developed by Ambrosio and Tortorelli $[2,3]$ for the Mumford-Shah model. This method has been extensively studied and extended for segmentation, inpainting, and several other applications in image analysis and processing (see, e.g., $[20,21,29,30,46])$. We propose a new multiphase segmentation model in the framework of $\Gamma$-convergence and phase transition, and develop the relevant mathematical analysis and computational strategies. More specifically, we propose to adopt the celebrated phasetransition model of Modica and Mortola [34] with a sinusoidal potential. The new model is a self-contained

${ }^{*}$ Jung and Shen are with the School of Mathematics, University of Minnesota, Minneapolis, MN 55455, (\{ymjung, jhshen\}@math.umn.edu); Kang is with the Department of Mathematics, University of Kentucky, Lexington, KY 40506, (skang@ms.uky.edu; the Corresponding Author). This research has been supported by NSF Program in Applied Mathematics under the grant numbers DMS-0202565 and DMS-0312223, as well as by the long term visiting program of IMA at the University of Minnesota. 
segmentation model, and is different from Ambrosio and Tortorelli's formulation [2], which approximates and computes the Mumford-Shah model.

We hereby emphasize that the similarities are inherent between image segmentation and the phase transition problem in material sciences and fluid mechanics. First of all, different phases in material sciences are characterized by the few key phase variables such as densities and tensions (e.g., ice versus water), while in image and vision analysis, distinct "object" segments are similarly characterized by some key visual features such as intensities, orientations, or more general Gabor features (e.g., in texture segmentation [42], [14]). Secondly, the difficulties in dealing with sharp interfaces emerging from both material phase transitions and image segmentation share the very same roots - the characteristic complexities in handling free boundaries and their geometry. Under such observations, it is beneficial for the imaging community to borrow the successful ideas in contemporary material sciences, e.g., the diffuse-interface model of Cahn-Hilliard [7], and its rigorous mathematical analysis in the framework of $\Gamma$-convergence approximation by Modica and Mortola [34] (as initially conjectured by De Giorgi).

Finally, as for all the major segmentation efforts in existence, the phase-field based segmentation model proposed herein is also nonlinear and nonconvex, and its robust computation (for local minima) is nontrivial. In the current work, we employ the so-called convex-concave (splitting) procedure (CCCP) as in the literatures of both computational nonlinear PDEs [4, 22, 53] and neural computation [54], and develop the corresponding computational schemes for the proposed energy functional.

The paper has been organized as follows. The new model is developed in Section 2. The relevant Mumford-Shah segmentation model and its related literature are briefly reviewed in Subsection 2.1, and the proposed Modica-Mortola sine-sinc model is established in Subsection 2.2. We analyze the major mathematical properties of the model in Section 3, including the $\Gamma$-convergence behavior in Subsection 3.2 and the existence and compactness theorems in Subsection 3.3. Computational schemes are presented in Section 4, where we develop the convex-splitting or the CCCP algorithm in Subsection 4.2, and demonstrate the numerical performance on generic image examples in Subsection 4.3. The conclusion is drawn in Section 5 .

\section{Multiphase Segmentation via Modica-Mortola Phase Transition}

In this section, we first motivate and develop the new model based upon the phase transition model of Modica and Mortola in material sciences and fluid dynamics [34], and discuss its connections to the Mumford-Shah segmentation model and some related works. Mathematical analysis will be further developed in the next section.

Let $\Omega$ be a bounded Lipschitz domain (e.g., a rectangle), and $u_{o}: \Omega \longrightarrow \mathbb{R}_{+} \cup\{0\}$ be a given image. First recall that the classical Mumford-Shah segmentation is to minimize the energy functional

$$
\mathcal{E}_{m s}\left[u, \Gamma \mid u_{o}\right]=\mathcal{H}^{1}(\Gamma)+\alpha \int_{\Omega \backslash \Gamma}|\nabla u|^{2} d x+\lambda \int_{\Omega}\left(u-u_{o}\right)^{2} d x
$$

where $\Gamma \in \Omega$ denotes the edge set of the ideal image $u$, and $\mathcal{H}^{1}$ represents the 1-dimensional Hausdorff measure. This functional is well defined on the admissible space:

$$
\mathcal{A}_{m s}=\left\{(u, \Gamma): u \in H^{1}(\Omega \backslash \Gamma), \mathcal{H}^{1}(\Gamma)<\infty, \Gamma \text { is relatively closed in } \Omega\right\},
$$

provided that the given image $u_{o} \in L^{2}(\Omega)$. In the language of machine learning [18, 41], $\mathcal{A}_{m s}$ represents the hypothesis space or model space of all piecewise smooth functions on $\Omega$.

\subsection{Piecewise constant segmentation model}

In order to identify individual objects, conceptually one has to carry out a post-processing step after the Mumford-Shah model outputs the edge set $\Gamma$. That is, one has to identify the individual connected components (or object patches) $\Omega_{i}$ 's of $\Omega \backslash \Gamma$. If each patch $\Omega_{i}$ is to be called a phase, then segmentation 
automatically bears the nature of multiple phases, since a generic image often contains multiple objects projected from the 3 -D world.

On the other hand, there also exist attempts to directly represent and compute different phases. Normally phase separation or identification relies upon the clustering of certain visual features such as frequency, local orientation, and local density, etc. A simple but commonly adopted phase feature is the image intensity value of a pixel. In the Mumford-Shah setting (1), this leads to the piecewise constant reduced Mumford-Shah (RMS) model,

$$
\mathcal{E}_{r m s}\left[u, \Gamma \mid u_{o}\right]=\mathcal{H}^{1}(\Gamma)+\lambda \int_{\Omega}\left(u-u_{o}\right)^{2} d x
$$

defined on the following admissible space,

$$
\mathcal{A}_{r m s}=\left\{(u, \Gamma):\left.D u\right|_{\Omega \backslash \Gamma}=0, \mathcal{H}^{1}(\Gamma)<\infty, \Gamma \text { is relatively closed in } \Omega\right\} \subseteq \mathcal{A}_{m s} .
$$

Here $D u$ denotes the vectorial Radon measure of the total variation (TV) of $u$.

The TV constraint requires any admissible $u$ to be constant on any connected component of $\Omega \backslash \Gamma$. For practical convenience, let us assume that there are finitely many such patches, say $K$ number of different patches. Then the entire image domain is partitioned to

$$
\Omega \backslash \Gamma=\bigcup_{k=0}^{K-1} \Omega_{k}
$$

On each patch $\Omega_{k}$, one must have $\left.u\right|_{\Omega_{k}}:=C_{k}, k=0, \ldots, K-1$ for a set of distinct intensity values $\mathbf{C}=\left(C_{0}, \ldots, C_{K-1}\right)$. Then the RMS energy $\mathcal{E}_{r m s}$ can be rewritten as

$$
\mathcal{E}_{r m s}\left[\mathbf{C}, \Gamma \mid u_{o}\right]=\frac{1}{2} \sum_{k=0}^{K-1} \mathcal{H}^{1}\left(\partial \Omega_{k}\right)+\lambda \sum_{k=0}^{K-1} \int_{\Omega_{k}}\left(C_{k}-u_{o}\right)^{2} d x .
$$

Notice that the factor $\frac{1}{2}$ is due to the double counting by any two adjacent patches. With successful level-set implementation, this reduced Mumford-Shah model has also been frequently referred to as the Chan-Vese model, honoring its rediscovery from the viewpoint of robust active contours in their well known paper [15].

The main mechanism of the proposed model is also to identify multiple phases by piecewise constant values. As already mentioned above, the core idea of our approach is unsurprisingly similar to those considered in $[17,28,49]$. But there are two major differences:

(i) All the aforementioned prior works are in essence still built upon the framework of Mumford and Shah (or its reduced form as discussed above), while our proposed model is not strictly a Mumford-Shah type model (though some equivalence will be established immediately below).

(ii) All the aforementioned prior works have employed the celebrated level-set technology of Osher and Sethian [39], while our new model adopts the phase field framework in material sciences and fluid mechanics. A level set function offers remarkable efficiency and robustness for representing and computing free boundaries, yet (strictly speaking) does not participate in the modeling process, while a phase field function is indispensably part of the model itself.

To proceed, we label each phase component with an integer, and define a signature function $z$ by

$$
z(x)=k, \text { if } x \in \Omega_{k}, \quad k=0, \ldots, K-1 .
$$

In practice, phase extraction is of course the very opposite process from getting the signature function $z$. That is, one has to first obtain the signature function $z$ before different phase patches $\Omega_{k}$ can be identified and extracted. For convenience, we shall also call $z$ a phase field. We then propose the multiphase segmentation model in this ideal scenario by minimizing

$$
E\left[\mathbf{C}, z \mid u_{o}\right]=\int_{\Omega}|D z(x)|+\lambda \sum_{k=0}^{K-1} \int_{\Omega}\left(C_{k}-u_{o}\right)^{2} \chi_{\{z=k\}} d x .
$$


As in the reduced Mumford-Shah or the Chan-Vese model $[13,36]$, the intensity distribution variable $\mathbf{C}$ can be conditionally solved by : from any given phase field $z$,

$$
C_{k}=\left\langle u_{o}\right\rangle_{\Omega_{k}}=\frac{1}{\left|\Omega_{k}\right|} \int_{\Omega_{k}} u_{o}(x) d x, \quad i=0, \ldots, K-1 .
$$

Then the ideal model only depends on the phase field $z$ :

$$
E\left[z \mid u_{o}\right]=\int_{\Omega}|D z(x)|+\lambda \sum_{k=0}^{K-1} \int_{\Omega}\left(C_{k}-u_{o}\right)^{2} \chi_{\{z=k\}} d x .
$$

The admissible class for the $i d e a l$ phase field is simply $\mathcal{A}=\{z \in B V(\Omega): z(x) \in \mathbb{Z}$ a.e. $\}$.

The main challenge of the proposed model arises from its mixture of the continuous TV Radon measure and the discrete constraint. Thus in the next subsection, this ideal model will be further polished in the framework of phase transitions and $\Gamma$-convergence.

Here we first emphasize that this ideal energy is somewhat equivalent to the piecewise constant MumfordShah functional. With the help of the signature function and the formulae for $C_{k}$ 's, $\mathcal{E}_{r m s}$ only depends on $\Gamma$ or $z$ and can be rewritten as

$$
\mathcal{E}_{r m s}\left[\Gamma \mid u_{o}\right]=\frac{1}{2} \sum_{k=0}^{K-1} \mathcal{H}^{1}(\partial\{z=k\})+\lambda \sum_{k=0}^{K-1} \int_{\Omega}\left(C_{k}-u_{o}\right)^{2} \chi_{\{z=k\}} d x .
$$

For any fixed number of phases $K$, the two functionals (3) and (4) are then equivalent, in the sense of

$$
\mathcal{E}_{r m s}\left[\Gamma \mid u_{o}\right] \leq E\left[z \mid u_{o}\right] \leq K \mathcal{E}_{r m s}\left[\Gamma \mid u_{o}\right]
$$

since

$$
\int_{\Omega}|D z|=\int_{\Gamma}|[z]| d \mathcal{H}^{1} \quad \text { and } \quad 1 \leq|[z]|<K .
$$

In most applications (especially in medical imaging), for instance, $K \leq 5$.

The difference between the two functionals (3) and (4) is also obvious, since the former weighs the jumps while the latter does not. For example, consider a rectangular domain $\Omega$ and two disjoint disks $\Omega_{1}, \Omega_{2}$ in $\Omega$ with radius $1 / 2$. If we assign $z=0$ on $\Omega_{0}=\Omega \backslash\left(\Omega_{1} \cup \Omega_{2}\right), z=1$ on $\Omega_{1}$ and $z=2$ on $\Omega_{2}$, then $\sum_{k=0}^{2} \mathcal{H}^{1}\left(\partial \Omega_{k}\right)=\frac{1}{2} \sum_{k=0}^{2} \mathcal{H}^{1}(\partial\{z=k\})=\frac{1}{2}(2 \pi+\pi+\pi)=2 \pi$, but $\int_{\Omega}|D z(x)|=1 \pi+2 \pi=3 \pi$. Thus, $\int_{\Omega}|D z|$ is a weighted length of $\Gamma$. Recall that the total variation Radon measure can be decomposed into:

$$
D z=\nabla z+\left.[z]\right|_{S_{z}} \mathcal{H}^{1}\left\llcorner S_{z}+\mathcal{C}_{z}\right.
$$

corresponding to the Lebesgue continuous gradient, the jump set $S_{z}=\Gamma$ (or reduced boundary) with $[z]=z^{+}-z^{-}$, and the singular Cantor measure. Now if the signature $z$ is ideally piecewise constant, both the Lebesgue and Cantor components must vanish, and one has

$$
\int_{\Omega}|D z|=\int_{S_{z}}|[z]| d \mathcal{H}^{1}
$$

which clearly shows the weighing nature of $\int_{\Omega}|D z|$.

Weighing the object boundaries certainly makes the proposed ideal model depending upon the labels. But for a fixed number $K$ of phases, the energies are more or less equivalent as just discussed. More importantly, it allows us to invoke the celebrated phase-transition approach in material sciences and fluid dynamics, in order to successfully overcome the major challenge in reconciling the two very opposite characteristics of the segmentation problem: continuum vs. discreteness. 


\subsection{The sine-sinc model via Modica-Mortola phase transition}

The objective functional (3)

$$
E\left[z \mid u_{o}\right]=\int_{\Omega}|D z(x)|+\lambda \sum_{k=0}^{K-1} \int_{\Omega}\left(C_{k}-u_{o}\right)^{2} \chi_{\{z=k\}} d x
$$

itself does not appear too complicated but becomes immensely baffling under the discrete constraint $z \in \mathbb{Z}$. This is a very common scenario in integer or discrete programming. For example, it is difficult to minimize this functional by any ordinary PDE approaches such as Euler-Lagrange equations or gradient-descent time marching.

We thus introduce its relaxed version via the celebrated model of Modica and Mortola [34] on phase transitions in material sciences and fluid mechanics. Recall that in the classical literature on phase transitions, the mixture of two immiscible and incompressible fluids are often modelled so that in equilibrium they separate into two phases with a minimal interface area. Cahn and Hilliard [7] first proposed to use a thin layer of continuous interface (i.e., diffuse interface) to model this separation. Later on Modica [33] proved that the Cahn-Hilliard model $\Gamma$-converges to the classical model. In another well known paper [34], Modica and Mortola established that the diffuse-interface energy

$$
F_{\epsilon}[z]=\int_{\Omega}\left[\epsilon|\nabla z|^{2}+\frac{1}{\epsilon} \sin ^{2} \pi z\right] d x, \quad \Gamma \text {-converges to } \frac{4}{\pi} \int_{\Omega}|D z(x)|,
$$

for phase fields that ultimately only take integer values. Some details will be further discussed in Section 3 .

Notice that in the Modica-Mortola model, the discrete constraint $z \in \mathbb{Z}$ has been softly enforced due to the sine potential regulated by the transition bandwidth $\epsilon$ in the denominator. In the present work, we adopt this Modica-Mortola diffuse-interface energy $F_{\epsilon}$ to approximate the ideal total variation energy in $E\left[z \mid u_{o}\right]$. This model thus well integrates both the TV regularity and the integer constraint $z \in \mathbb{Z}$.

For the data-fitting term, instead of the ideal indicator $\chi_{\{z=k\}}$ or Kronecker's delta, we also propose to use a properly relaxed version to facilitate model analysis and computing. More specifically, we choose $\operatorname{sinc}^{2}(z-k)$ to match the sine potential in Modica and Mortola's phase transition energy,

$$
G\left[z \mid u_{o}\right]=\sum_{k=0}^{K-1} \int_{\Omega}\left|C_{k}-u_{o}\right|^{2} \operatorname{sinc}^{2}(z-k) d x .
$$

Recall that the sinc function is defined as $\operatorname{sinc}(z)=\frac{\sin \pi z}{\pi z}$ for $z \in \mathbb{R}$. For a phase field $z$ that almost only takes integer values, sinc leads to desirable approximations to the indicator functions of integer phases and is more appealing computationally. This is because (i) $\operatorname{sinc}(k)=\delta_{k}$ (Kronecker's delta) for $k \in \mathbb{Z}$, the so-called interpolating property in the celebrated theorem of Shannon interpolation $[12,19,48]$; and (ii) $\operatorname{sinc}(z)$ is an entire function for $z \in \mathbb{C}$, and when $z \in \mathbb{R}$,

$$
\frac{d}{d z} \operatorname{sinc}(z)=\mathrm{O}\left(\frac{1}{|z|}\right), \quad \text { as } z \rightarrow \pm \infty .
$$

Thus in particular, $\operatorname{sinc}(z) \in W^{1, \infty}(\mathbb{R})$ and is Lipschitz continuous. As a result, the sinc-approximation will facilitate both analysis and computation later on.

In combination, we have arrived at the relaxed version of the ideal multiphase segmentation model (3) with a given number $K$ of phases:

$$
\begin{aligned}
E_{\epsilon}\left[z \mid u_{o}\right] & =F_{\epsilon}[z]+\lambda G\left[z \mid u_{o}\right] \\
& =\int_{\Omega}\left[\epsilon|\nabla z|^{2}+\frac{1}{\epsilon} \sin ^{2} \pi z\right] d x+\lambda \sum_{k=0}^{K-1} \int_{\Omega}\left|C_{k}-u_{o}\right|^{2} \operatorname{sinc}^{2}(z-k) d x .
\end{aligned}
$$


Here the values $C_{k}$ 's have been conditionally optimized by the following equation with any given phase field $z$ (under the least-square principle):

$$
C_{k}=C_{k}[z]= \begin{cases}\frac{\int_{\Omega} u_{o} \operatorname{sinc}^{2}(z-k) d x}{\int_{\Omega} \operatorname{sinc}^{2}(z-k) d x} & \text { if } \int_{\Omega} \operatorname{sinc}^{2}(z-k) d x>0, \\ 0 & \text { otherwise. }\end{cases}
$$

When $z(x)$ only takes integer values, this $C_{k}$ indeed reproduce the value introduced in (2). If the denominator in (6) vanishes, since

$$
\int_{\Omega} \operatorname{sinc}^{2}(z-k) d x=0 \Longleftrightarrow \operatorname{sinc}(z(x)-k)=0, \text { a.e } x \in \Omega \Longleftrightarrow z(x) \in \mathbb{Z} \backslash\{k\},
$$

we observe that the phase $k$ is empty and redundant. Also in this case the particular value of $C_{k}[z]$ is unimportant, since the integral $\int_{\Omega}\left|u-C_{k}\right|^{2} \operatorname{sinc}^{2}(z-k) d x$ vanishes.

\section{3 Г-Convergence of the Model and Existence of Minimizers}

In this section, we develop the necessary analysis of the proposed model. Following a brief review of the $\Gamma$-convergence theory, we first show that the relaxed model (5) converges to the original piecewise constant model (3), and then prove that optimal segmentation does exist for any fixed $\epsilon$. Compactness of the minimizers for all $\epsilon$ 's is also discussed in the end.

\subsection{Brief review of $\Gamma$-convergence}

$\Gamma$-convergence was first introduced by De Giorgi and Franzoni in [24] to facilitate analysis and approximation of PDEs and variational problems. Since then it has been widely applied to phase transition models in material sciences, the modeling of thin films or plates, homogenization of variational problems, as well as free discontinuity problems (e.g., [6, 31]). In image processing, the most influential application is Ambrosio-Tortorelli's $\Gamma$-convergence approximation to the Mumford-Shah functional [2]. The definition of $\Gamma$-convergence is as follows.

Definition 1 ( $\Gamma$-convergence) Let $X$ be a metric space and $\mathcal{F}_{\epsilon}: X \rightarrow \overline{\mathbb{R}}$ for $\epsilon>0$. We say that $\mathcal{F}_{\epsilon}$ $\Gamma$-converges to $\mathcal{F}$ in $X$ as $\epsilon \rightarrow 0$ and write $\mathcal{F}_{\epsilon} \stackrel{\Gamma}{\rightarrow} \mathcal{F}$, if the following two conditions holds for all $u \in X$ :

(i) (liminf inequality) for every sequence $\left(u_{\epsilon}\right)$ converging to $u$,

$$
\mathcal{F}(u) \leq \liminf _{\epsilon \rightarrow 0} \mathcal{F}_{\epsilon}\left(u_{\epsilon}\right)
$$

(ii) (limsup inequality) there exists a sequence $\left(u_{\epsilon}\right)$ converging to $u$ such that

$$
\mathcal{F}(u) \geq \limsup _{\epsilon \rightarrow 0} \mathcal{F}_{\epsilon}\left(u_{\epsilon}\right) .
$$

The most important properties of $\Gamma$-convergence are summarized by the following theorem. We refer the reader to $[1,6,31]$ for further discussion and deeper development.

Theorem $1 \Gamma$-convergence have the following properties.

(i) (Spatial stability of the limit) the $\Gamma$-limit $\mathcal{F}$ is lower semicontinuous;

(ii) (Stability under continuous perturbations) if $\mathcal{F}_{\epsilon} \stackrel{\Gamma}{\rightarrow} \mathcal{F}$ and $\mathcal{G}$ is continuous, then $\mathcal{F}_{\epsilon}+\mathcal{G} \stackrel{\Gamma}{\rightarrow} \mathcal{F}+\mathcal{G}$; 
(iii) (Stability of minimizing sequences) if $\mathcal{F}_{\epsilon} \stackrel{\Gamma}{\rightarrow} \mathcal{F}$ and $v_{\epsilon}$ minimizes $\mathcal{F}_{\epsilon}$, then every cluster point of $\left(v_{\epsilon}\right)$ minimizes $\mathcal{F}$.

Let us briefly comment on these three properties. Property $(i)$ reveals the necessary condition for designing $\Gamma$-convergence approximation to a target functional. Property $(i i)$ paves the way to extending existing $\Gamma$ convergence schemes, which is particularly helpful in the current work. Property (iii) reveals the real essence of the entire machinery of $\Gamma$-convergence, by which the minimization of a touchy objective $\mathcal{F}$ is tamed or relaxed by a family of better behaved objectives $\mathcal{F}_{\epsilon}$.

Directly benefiting the current work is the following remarkable theorem in the original paper of ModicaMortola [34], which historically has played significant roles in the theories of $\Gamma$-convergence, phase transitions, and the Cahn-Hilliard model.

Theorem 2 (Modica and Mortola [34]) Define $\mathcal{S}=\left\{z \in B V\left(\mathbb{R}^{n}\right): z(x) \in \mathbb{Z}\right.$, a.e. $\left.x\right\}$, and

$$
\begin{aligned}
& F_{\epsilon}(z):= \begin{cases}\int_{\mathbb{R}^{n}}\left[\epsilon|\nabla z(x)|^{2}+\frac{1}{\epsilon} \sin ^{2}(\pi z(x))\right] d x, & \text { for } z \in H^{1}\left(\mathbb{R}^{n}\right) \cap L^{1}\left(\mathbb{R}^{n}\right) \\
+\infty, & \text { for } z \in L^{1}\left(\mathbb{R}^{n}\right) \backslash H^{1}\left(\mathbb{R}^{n}\right),\end{cases} \\
& F(z):= \begin{cases}\frac{4}{\pi} \int_{\mathbb{R}^{n}}|D z(x)| & \text { for } z \in S\left(\mathbb{R}^{n}\right), \\
+\infty & \text { for } z \in L^{1}\left(\mathbb{R}^{n}\right), \text { but } z \notin S\left(\mathbb{R}^{n}\right) .\end{cases}
\end{aligned}
$$

Then, the functional $F_{\epsilon} \Gamma$-converges to $F$ as $\epsilon \rightarrow 0$ in $L^{1}\left(\mathbb{R}^{n}\right)$.

We note that the domain $\mathbb{R}^{n}$ can be replaced by any regular open bounded domain $\Omega$, which is the case in the current application. Theorem 2 was originally conjectured by De Giorgi and then proven by Modica and Mortola [34] in 1977, shortly after the notion of $\Gamma$-convergence was introduced in [24]. The connection with the Cahn-Hilliard model was established in Modica [33].

\section{$3.2 \quad \Gamma$-convergence of the sine-sinc model}

In image processing, the image range is often bounded by $u_{o} \in[0,1]$ in the analogue setting and $u_{o} \in[0,255]$ in the digital setting with 8 bits. Therefore, by default we shall assume $u_{o} \in L^{\infty}(\Omega)$ for maximal technical clarity.

In the Modica-Mortola sine-sinc model proposed in (5),

$$
\begin{aligned}
E_{\epsilon}\left[z \mid u_{o}\right] & =F_{\epsilon}[z]+\lambda G\left[z \mid u_{o}\right] \\
& =\int_{\Omega}\left[\epsilon|\nabla z|^{2}+\frac{1}{\epsilon} \sin ^{2} \pi z\right] d x+\lambda \sum_{k=0}^{K-1} \int_{\Omega}\left|C_{k}-u_{o}\right|^{2} \operatorname{sinc}^{2}(z-k) d x,
\end{aligned}
$$

the first term $F_{\epsilon}$ has already been proven to $\Gamma$-converge to $F$ in [34]. In this subsection, we show that the fitting term $G$ is continuous in $L^{1}(\Omega)$.

In what follows, we shall use the notation $\omega(x \mid z)$ to denote a spatial function $\omega(\cdot \mid z)$ that depends on the given phase field $z=z(x)$. Such dependence could be local in the form of $g(x, z(x))$, or global in the form of $g(x, J[z])$ where $J[z]$ is a functional on $z$. The following general theorem gives a unified foundation for the proof of $\Gamma$-convergence of the proposed model.

\section{Theorem 3 Suppose that}

(i) $\varphi: \mathbb{R} \rightarrow \mathbb{R}$ is Lipschitz continuous with $|\varphi(x)-\varphi(y)| \leq L|x-y|$,

(ii) $\left\|w\left(\cdot \mid z_{n}\right)-w(\cdot \mid z)\right\|_{L^{\infty}(\Omega)} \rightarrow 0$ as $\left\|z_{n}-z\right\|_{L^{1}(\Omega)} \rightarrow 0$,

(iii) $\|w(\cdot \mid z)\|_{L^{\infty}(\Omega)} \leq M$ for some positive $M$, for all $z \in L^{1}(\Omega)$, 
then, $g[z]=\int_{\Omega} w(x \mid z) \varphi(z) d x$ is continuous for $z \in L^{1}(\Omega)$.

Proof. Let $\left\{z_{n}\right\}$ be a sequence converging to $z: z_{n} \rightarrow z$ in $L^{1}(\Omega)$. Then

$$
\begin{aligned}
\mid g\left[z_{n}\right]- & g[z] \mid \\
& \leq\left|\int_{\Omega} w\left(x \mid z_{n}\right) \varphi\left(z_{n}\right)-w(x \mid z) \varphi\left(z_{n}\right) d x\right|+\left|\int_{\Omega} w(x \mid z) \varphi\left(z_{n}\right)-w(x \mid z) \varphi(z) d x\right| \\
& \leq\left\|w\left(x \mid z_{n}\right)-w(x \mid z)\right\|_{L^{\infty}(\Omega)}\left\|\varphi\left(z_{n}\right)\right\|_{L^{1}(\Omega)}+M \int_{\Omega}\left|\varphi\left(z_{n}\right)-\varphi(z)\right| d x \\
& \leq L\left\|z_{n}\right\|_{L^{1}(\Omega)}\left\|\omega\left(x \mid z_{n}\right)-w(x \mid z)\right\|_{L^{\infty}(\Omega)}+M L\left\|z_{n}-z\right\|_{L^{1}(\Omega)} .
\end{aligned}
$$

Both terms tend to zero as $\left\|z_{n}-z\right\|_{L^{1}(\Omega)} \rightarrow 0$ by the assumption (ii).

This theorem can be applied to establish that $C_{k}[z]$ 's are continuous, and eventually that $G\left[z \mid u_{o}\right]$ is continuous as well.

Corollary 1 Suppose $z_{n} \rightarrow z$ in $L^{1}$ and $\int_{\Omega} \operatorname{sinc}^{2}(z(x)-k) d x \neq 0$. Then $C_{k}(z)$ defined in (6) is continuous in $z$, i.e., $C_{k}\left[z_{n}\right] \rightarrow C_{k}[z]$.

This is directly proven by Theorem 3 with $w(x)=u_{o}(x)$ (for the denominator) and $w(x)=1$ (for the numerator), as well as $\varphi(z)=\sin ^{2}(z(x)-k)$.

Corollary 2 (Degenerate Case) If $\varphi(z(x))=0$ a.e., then, $g[z]=0$ and condition (ii) in Theorem 3 can be dropped.

It simply comes from the definition of $g[z]$, and the conditions (i) and (iii):

$$
\left|g\left[z_{n}\right]\right| \leq M \int_{\Omega}\left|\varphi\left(z_{n}\right)\right|=M \int_{\Omega}\left|\varphi\left(z_{n}\right)-\varphi(z)\right| d x \leq M L \int_{\Omega}\left|z_{n}-z\right| d x \rightarrow 0 .
$$

Proposition 1 The fitting functional $G$ is continuous in $L^{1}(\Omega)$ :

$$
G\left[z \mid u_{o}\right]=\sum_{k=0}^{K-1} \int_{\Omega}\left|C_{k}-u_{o}\right|^{2} \operatorname{sinc}^{2}(z-k) d x
$$

with

$$
C_{k}=C_{k}[z]= \begin{cases}\frac{\int_{\Omega} u_{o} \operatorname{sinc}^{2}(z-k) d x}{\int_{\Omega} \operatorname{sinc}^{2}(z-k) d x}, & \text { if } \int_{\Omega} \operatorname{sinc}^{2}(z-k) d x>0 \\ 0, & \text { otherwise. }\end{cases}
$$

Proof. Let $w(x \mid z)=\left(u_{o}(x)-C_{k}[z]\right)^{2}$ and $\varphi(z)=\operatorname{sinc}^{2}(z-k)$ for $z \in L^{1}(\Omega)$. We now show that $G\left[z \mid u_{o}\right]$ indeed satisfies all three conditions of Theorem 3. Condition (i) is clear since $\varphi$ is Lipschitz continuous. For (ii), let $\left\{z_{n}\right\}$ be a converging sequence $z_{n} \rightarrow z$ in $L^{1}(\Omega)$. Then

$$
\begin{aligned}
\left|w\left(x \mid z_{n}\right)-w(x \mid z)\right| & =\left|\left(C_{k}\left[z_{n}\right]-u_{o}(x)\right)^{2}-\left(C_{k}[z]-u_{o}(x)\right)^{2}\right| \\
& \leq\left|C_{k}\left[z_{n}\right]+C_{k}[z]-2 u_{o}(x)\right|\left|C_{k}\left[z_{n}\right]-C_{k}[z]\right| \\
& \leq 4\left\|u_{o}\right\|_{L^{\infty}(\Omega)}\left|C_{k}\left[z_{n}\right]-C_{k}[z]\right|,
\end{aligned}
$$

and $\left|C_{k}\left[z_{n}\right]-C_{k}[z]\right| \rightarrow 0$ from the continuity of $C_{k}$ in Corollary 1. Thus $\left|w\left(x \mid z_{n}\right)-w(x \mid z)\right| \rightarrow 0$. For (iii), notice that $\left|C_{k}[z]\right| \leq\left\|u_{o}\right\|_{L^{\infty}(\Omega)}$ for $\forall z$. Then,

$$
w(x \mid z) \leq\left(\|u\|_{L^{\infty}(\Omega)}+\left|C_{k}[z]\right|\right)^{2} \leq\left(2\left\|u_{o}\right\|_{L^{\infty}(\Omega)}\right)^{2} \text { for all } x \in \Omega .
$$

Therefore, by Theorem $3, G\left[z \mid u_{o}\right]$ is continuous in $L^{1}(\Omega)$.

Finally, the $\Gamma$-convergence behavior of the proposed Modica-Mortola sine-sinc model becomes evident from the combination of Proposition 1, Theorem 2, and Theorem 1.

Theorem 4 ( $\Gamma$-Convergence) $E_{\epsilon} \Gamma$-converges to $E$ w.r.t. the $L^{1}(\Omega)$ topology. 


\subsection{Existence of minimizers of $E_{\epsilon}$}

In this subsection we show that for each $\epsilon$, a minimizer to $E_{\epsilon}$ does exist. We shall also briefly discuss the compactness of a sequence of minimizers for all $\epsilon$ 's.

Theorem 5 (Existence of Minimizers) Suppose the given image $u_{o} \in L^{2}(\Omega)$ and denote the admissible space for $K$-phase segmentation by:

$$
\mathcal{A}_{K}=\left\{z \in H^{1}(\Omega):-1 / 2<z<K-1 / 2\right\} .
$$

Then for each $\epsilon>0$, there exists a minimizer of $E_{\epsilon}$ in $\mathcal{A}_{K}$.

Proof. We first show that the infimum is finite. Consider the uniform phase $z \equiv 0 \in \mathcal{A}_{K}$. Then the Modica-Mortola energy $F_{\epsilon}[z]=0$, and $C_{0}=\frac{1}{|\Omega|} \int_{\Omega} u_{o}(x) d x<\infty$ while $C_{k}=0$ for $k=1, \ldots, K-1$. Therefore,

$$
E_{\epsilon}\left[0 \mid u_{o}\right]=\lambda \int_{\Omega}\left|C_{0}-u_{o}(x)\right|^{2} d x<\infty
$$

and since $E_{\epsilon}\left[\cdot \mid u_{o}\right] \geq 0$, the claim is proved.

Let $\left\{z_{n}\right\}$ be a minimizing sequence for $E_{\epsilon}$ in $\mathcal{A}_{K}$. Since $\sup _{n} \int_{\Omega}\left|\nabla z_{n}\right|^{2} d x<\infty$ from the Modica-Mortola energy, as well as $-1 / 2<z_{n}<K-1 / 2$, the sequence $\left\{z_{n}\right\}$ must be precompact in $L^{1}(\Omega)$ by RellichKondrachov's compactness theorem. There thus exists a subsequence of $\left\{z_{n}\right\}$, which is still denoted by $\left\{z_{n}\right\}$ after relabelling to simplify notations, such that

$$
z_{n} \rightarrow z^{*} \text { in } L^{2}(\Omega), \text { for some } z^{*} \in L^{2}(\Omega) .
$$

As a result, one has the weak convergence for the gradient fields:

$$
\nabla z_{n} \rightarrow \nabla z^{*} \text { weakly in } L^{2}(\Omega) .
$$

Then, by the lower semicontinuity of the $L^{2}$-norm under the weak topology,

$$
\int_{\Omega}\left|\nabla z^{*}\right|^{2} d x \leq \liminf _{n \rightarrow \infty} \int_{\Omega}\left|\nabla z_{n}\right|^{2} d x
$$

For the other two terms with sine and sinc functions, convergence follows from Lebesgue's dominated convergence theorem (LDCT) as shown below. First, possibly with another round of subsequence refinement and relabelling, one can further assume that $z_{n} \rightarrow z^{*}$ a.e. Then, $\sin ^{2} \pi z_{n} \rightarrow \sin ^{2} \pi z^{*}$ a.e., and by LDCT,

$$
\int_{\Omega} \sin ^{2} \pi z_{n} d x \rightarrow \int_{\Omega} \sin ^{2} \pi z^{*} d x
$$

Similarly, $\operatorname{sinc}^{2} \pi\left(z_{n}-k\right) \rightarrow \operatorname{sinc}^{2} \pi\left(z^{*}-k\right)$ a.e. for $k=0, \ldots, K-1$. By LDCT, one has $\int_{\Omega} \operatorname{sinc}^{2} \pi\left(z_{n}-k\right) d x \rightarrow$ $\int_{\Omega} \operatorname{sinc}^{2} \pi\left(z^{*}-k\right) d x$, and

$$
\int_{\Omega} u_{o}(x) \operatorname{sinc}^{2} \pi\left(z_{n}-k\right) d x \rightarrow \int_{\Omega} u_{o}(x) \operatorname{sinc}^{2} \pi\left(z^{*}-k\right) d x
$$

since $u_{o} \in L^{2}(\Omega) \subset L^{1}(\Omega)$. Consequently, if for the $k$-th phase $\int_{\Omega} \operatorname{sinc}^{2} \pi\left(z^{*}-k\right) d x>0$, then $\left(C_{k}\left[z_{n}\right]\right)_{n}$ must be a bounded sequence. Since $u_{o} \in L^{2}(\Omega)$, by LDCT, as $n \rightarrow \infty$,

$$
\int_{\Omega}\left|u_{o}-C_{k}\left[z_{n}\right]\right|^{2} \operatorname{sinc}^{2} \pi\left(z_{n}-k\right) d x \rightarrow \int_{\Omega}\left|u_{o}-C_{k}\left[z^{*}\right]\right|^{2} \operatorname{sinc}^{2} \pi\left(z^{*}-k\right) d x .
$$

If on the other hand, $\operatorname{sinc}^{2} \pi\left(z^{*}-k\right)=0$ a.e., then

$$
\int_{\Omega}\left|u_{o}-C_{k}\left[z^{*}\right]\right|^{2} \operatorname{sinc}^{2}\left(z^{*}-k\right) d x=0 \leq \liminf _{n \rightarrow \infty} \int_{\Omega}\left|u_{o}-C_{k}\left[z_{n}\right]\right|^{2} \operatorname{sinc}^{2}\left(z^{*}-k\right) d x .
$$


Finally, in combination of (8), (9), (10) and (11), we have

$$
E_{\epsilon}\left[z^{*} \mid u_{o}\right] \leq \liminf _{n \rightarrow \infty} E_{\epsilon}\left[z_{n} \mid u_{o}\right] \leq \inf _{z \in \mathcal{A}_{K}} E_{\epsilon}\left[z \mid u_{o}\right]
$$

and the limit $z^{*}$ has to be a minimizer of $E_{\epsilon}$. This completes the proof.

Notice that in this theorem, we have even allowed the given image $u_{o} \in L^{2}(\Omega)$, instead of $u_{o} \in L^{\infty}(\Omega)$, which is the default assumption throughout the work.

Finally we briefly comment on the compactness or stability of the sequence of minimizers from the $\Gamma$-convergence approximation.

Theorem 6 (Compactness of the Sequence of Minimizers) Following the preceding theorem, let $z_{\epsilon}$ minimizes $E_{\epsilon}$ for each $\epsilon>0$. Then there exists a subsequence $\left(z_{\epsilon^{\prime}}\right)$ of $\left(z_{\epsilon}\right)$ and some $z \in L^{1}(\Omega)$ such that $z_{\epsilon^{\prime}} \longrightarrow z$ in $L^{1}(\Omega)$ as $\epsilon^{\prime} \rightarrow 0$, and $z$ minimizes $E$.

Proof. By the Cauchy-Schwarz inequality,

$$
\begin{aligned}
E_{\epsilon}\left[z \mid u_{o}\right] & \geq F_{\epsilon}[z]=\int_{\Omega}\left[\epsilon|\nabla z|^{2}+\frac{1}{\epsilon} \sin ^{2} \pi z\right] d x \\
& \geq 2 \int_{\Omega}|\nabla z||\sin \pi z| d x=\int_{\Omega}|\nabla(H(z))| d x .
\end{aligned}
$$

Here $H:\left(-\frac{1}{2}, K-\frac{1}{2}\right) \rightarrow \mathbb{R}$ satisfies $H^{\prime}(r)=2|\sin \pi r|$ and $H(0)=0$. By $(7)$, there exists $M>0$ such that $E_{\epsilon}\left[z_{\epsilon} \mid u_{o}\right] \leq M$ for all $\epsilon$. By (12), the sequence of functions $\left(h_{\epsilon}(x)=H\left(z_{\epsilon}(x)\right)\right)_{\epsilon}$ must be bounded in $B V(\Omega)$, and thus precompact in $L^{1}(\Omega)$. By subsequence refinement, one can assume that there exists a subsequence $h_{\epsilon^{\prime}} \rightarrow h$ a.e. for some $h \in L^{1}(\Omega)$. Since $H(r)$ is continuous and strictly monotone, it admits a continuous inverse. Thus one has $z_{\epsilon^{\prime}}(x) \longrightarrow z(x)=H^{-1}(h(x))$, a.e. Since $z_{\epsilon} \in\left(-\frac{1}{2}, K-\frac{1}{2}\right)$ is uniformly bounded for $\epsilon$, one must have $z_{\epsilon^{\prime}} \rightarrow z$ in $L^{1}(\Omega)$ by LDCT. Then the rest of the theorem follows from Theorem 1 .

\section{Computation and Experiments}

In this section, we develop the computational schemes for the proposed model. The major difficulty arises from the fact that the Modica-Mortola sine-sinc functional is non-convex. In this paper, we apply the method of convex splitting or the concave-convex procedure (CCCP) for robustly computing the local minima of the model. After a brief review on the CCCP method, we detail our computational strategies, and test the schemes on some generic examples involving both synthetic and natural images.

\subsection{Review of the concave-convex procedure (CCCP)}

There have been growing interests recently in how to solve non-convex function efficiently. In [22] in the setting of gradient flows, Eyre proposed to split non-convex functions into two functions, one contractive and the other expansive. It included computational examples of the Cahn-Hilliard equation with different time steps. More analysis on the numerical algorithms for the Cahn-Hilliard or Allen-Cahn equations were studied by Vollmayr-Lee and Rutenberg in [53], where unconditionally stable time step was explored. The idea of convex splitting has also been recently applied to the Cahn-Hilliard inpainting by Bertozzi et al. [4].

Independent of the computational PDE literature, on the other hand, the similar idea of convex splitting was also explored by Yuille and Rangarajan [54] in a more general setting of neural computation, where the method has been termed the Convex-Concave Procedure (CCCP). The method has found many important applications in computer vision and neural computation.

Theorem 7 (Yuille and Rangarajan [54]) Let $\mathcal{E}(\vec{x})$ with $\vec{x} \in \mathbb{R}^{n}$ be an energy function with a bounded Hessian. Then it can be decomposed into the sum of a convex function and a concave function. 
Theorem 8 (Yuille and Rangarajan [54]) Consider an energy function which is bounded below and is an addition of convex and concave functions:

$$
\mathcal{E}(\vec{x})=\mathcal{E}_{\text {convex }}(\vec{x})+\mathcal{E}_{\text {concave }}(\vec{x}) .
$$

Then, the discrete iterative CCCP algorithm given by

$$
\nabla \mathcal{E}_{\text {convex }}\left(\vec{x}^{n+1}\right)=-\nabla \mathcal{E}_{\text {concave }}\left(\vec{x}^{n}\right), \quad n=0,1, \cdots
$$

is guaranteed to monotonically decrease the energy $\mathcal{E}(\vec{x})$ as a function of time and to converge to a local minimum or a saddle point of $\mathcal{E}(\vec{x})$.

We briefly comment on these results for our application. First, notice that (13) is only solvable when

$$
\operatorname{Range}\left(-\nabla \mathcal{E}_{\text {concave }}\right) \subseteq \operatorname{Range}\left(\mathcal{E}_{\text {convex }}\right)
$$

In particular, the condition holds when $\operatorname{Range}\left(\mathcal{E}_{\text {convex }}\right)=\mathbb{R}^{n}$, the entire space. The solvability condition (14) implies that, in some sense, the convex part must be stronger than or dominant over the concave part, which is often (practically) true for energy minimization problems when the function $f$ is bounded below and $f \rightarrow \infty$ as $|\vec{x}| \rightarrow \infty$.

Secondly, in the present context, the CCCP should be applied to the functional setting instead of the function in $\mathbb{R}^{n}$. Therefore the gradients in (13) should be naturally replaced by the Fréchet derivatives of the functionals. In our application, the functional is indeed Fréchet differentiable. (In general, the CCCP iteration (13) can also be based upon the sub-gradients of convex function(al)s since the splitting yields convex components.)

\subsection{Details of the computational scheme}

To compute the optimization problem (5),

$$
\begin{aligned}
E_{\epsilon}\left[z \mid u_{o}\right] & =F_{\epsilon}[z]+\lambda G\left[z \mid u_{o}\right] \\
& =\int_{\Omega}\left[\epsilon|\nabla z|^{2}+\frac{1}{\epsilon} \sin ^{2} \pi z\right] d x+\lambda \sum_{k=0}^{K-1} \int_{\Omega}\left|C_{k}-u_{o}\right|^{2} \operatorname{sinc}^{2}(z-k) d x,
\end{aligned}
$$

we treat $\mathbf{C}=\left(C_{0}, \ldots, C_{K-1}\right)$ as an independent variable by breaking the dependency of $\mathbf{C}$ on the function $z$, and compute $E_{\epsilon}\left[z, \mathbf{C} \mid u_{o}\right]$ regarding $z$ and $\mathbf{C}$ as independent variables. This allows the application of the alternating minimization (AM) scheme, i.e., to alternatingly optimize the two conditional energies $E_{\epsilon}\left[z \mid \mathbf{C}, u_{o}\right]$ and $E_{\epsilon}\left[\mathbf{C} \mid z, u_{o}\right]$, under the iterations of $z^{n} \rightarrow \mathbf{C}^{n} \rightarrow z^{n+1}$ given by

$$
\begin{aligned}
& \mathbf{C}^{n}=\operatorname{argmin} E_{\epsilon}\left[\mathbf{C} \mid z^{n}, u_{o}\right], \\
& z^{n+1}=\operatorname{argmin} E_{\epsilon}\left[z \mid \mathbf{C}^{n}, u_{o}\right] .
\end{aligned}
$$

It is well known (i.e., Vogel [52] or Shen [44]) that the AM scheme is monotone:

$$
E_{\epsilon}\left[z^{n+1}, \mathbf{C}^{n+1} \mid u_{o}\right] \leq E_{\epsilon}\left[z^{n}, \mathbf{C}^{n} \mid u_{o}\right] .
$$

To minimize (15), one simply computes at the pixel level,

$$
C_{k}=\frac{\sum_{i} \sum_{j} u_{i, j} \operatorname{sinc}^{2}\left(z_{i, j}^{n}-k\right)}{\sum_{i} \sum_{j} \operatorname{sinc}^{2}\left(z_{i, j}^{n}-k\right)}, \quad k=0, \ldots, K-1,
$$

where $z_{i, j}^{n}$ denotes computational phase field on the Cartesian image domain.

We then apply the concave-convex procedure to minimize $E_{\epsilon}\left[z \mid \mathbf{C}^{n}, u_{o}\right]$ in (16). For convenience, we shall omit the superscript $n$ of $\mathbf{C}^{n}$ hereafter. First, we add simple convex functionals to express $E_{\epsilon}$ as the difference of two convex functionals. By noticing that if $f$ is a convex function from $\mathbb{R}$ to $\mathbb{R}$, then the functional $F(u)=\int_{\Omega} f(u(x)) d x$ is a convex functional, we have the following proposition. 
Proposition 2 Let $F(u)=\int_{\Omega} f(u(x)) d x$, where $f \in C^{2}(\mathbb{R})$ and $f^{\prime \prime} \geq-\gamma$ for some $\gamma \geq 0$. Define the splitting

$$
F(u)=\int_{\Omega}\left(f(u)+\frac{\gamma}{2} u^{2}\right) d x-\int_{\Omega} \frac{\gamma}{2} u^{2} d x:=F^{1}(u)-F^{2}(u) .
$$

Then both $F^{1}$ and $F^{2}$ are convex.

The proof is trivial since $f_{1}^{\prime \prime}(u) \geq 0$ if $f_{1}(u)=f(u)+\frac{\gamma}{2} u^{2}$. We now apply this splitting technique to the proposed model $E_{\epsilon}$ in (5).

We shall add two sets of terms, one for the non-convex Modica-Mortola functional $F_{\epsilon}$, and the other for the non-convex fitting term $G$. For the functional $F_{\epsilon}$, we add $\frac{\pi^{2}}{\epsilon} \int_{\Omega}|z|^{2} d x$ by noticing that $\frac{d^{2}}{d z^{2}} \sin ^{2} \pi z \geq-2 \pi^{2}$ :

$$
F_{\epsilon}[z]=\left(F_{\epsilon}[z]+\frac{\pi^{2}}{\epsilon} \int_{\Omega}|z|^{2} d x\right)-\frac{\pi^{2}}{\epsilon} \int_{\Omega}|z|^{2} d x:=F_{\epsilon}^{1}[z]-F_{\epsilon}^{2}[z]
$$

Similarly, the fitting term $G$ in (5) can be split into $G\left[z \mid \mathbf{C}, u_{o}\right]=G^{1}\left[z \mid \mathbf{C}, u_{o}\right]-G^{2}\left[z \mid \mathbf{C}, u_{o}\right]$, where

$$
\begin{gathered}
G^{1}\left[z \mid \mathbf{C}, u_{o}\right]=G\left[z \mid \mathbf{C}, u_{o}\right]+\frac{\pi^{2}}{3} \sum_{k=0}^{K-1} \int_{\Omega}\left|u_{o}-C_{k}\right|^{2}|z-k|^{2} d x \\
G^{2}\left[z \mid \mathbf{C}, u_{o}\right]=\frac{\pi^{2}}{3} \sum_{k=0}^{K-1} \int_{\Omega}\left|u_{o}-C_{k}\right|^{2}|z-k|^{2} d x
\end{gathered}
$$

since $\frac{d^{2}}{d z^{2}} \operatorname{sinc}^{2} z \geq \frac{-2 \pi^{2}}{3}$.

Thus, the functional (5) becomes $E_{\epsilon}=E_{\epsilon}^{1}-E_{\epsilon}^{2}=\left(F_{\epsilon}^{1}+\lambda G^{1}\right)-\left(F_{\epsilon}^{2}+\lambda G^{2}\right)$. We then apply the CCCP algorithm (13) via the Fréchet derivative:

$$
\left(F_{\epsilon}^{1}+\lambda G^{1}\right)^{\prime}\left(z^{n+1}\right)=\left(F_{\epsilon}^{2}+\lambda G^{2}\right)^{\prime}\left(z^{n}\right) .
$$

Under integration by parts, (18) is equivalent to the PDE:

$$
\begin{gathered}
{\left[-2 \epsilon \Delta z^{n+1}+\frac{\pi}{\epsilon} \sin 2 \pi z^{n+1}\right]+\frac{2 \pi^{2}}{\epsilon} z^{n+1}} \\
+\left[\lambda \sum_{k=0}^{K-1}\left|u_{o}-C_{k}\right|^{2} \frac{d}{d z} \operatorname{sinc}^{2}\left(z^{n+1}-k\right)\right]+\lambda \sum_{k=0}^{K-1}\left|u_{o}-C_{k}\right|^{2} \frac{2 \pi^{2}}{3}\left(z^{n+1}-k\right) \\
=\frac{2 \pi^{2}}{\epsilon} z^{n}+\lambda \sum_{k=0}^{K-1}\left|u_{o}-C_{k}\right|^{2} \frac{2 \pi^{2}}{3}\left(z^{n}-k\right) .
\end{gathered}
$$

Here the terms in the square brackets come from the Euler-Lagrange equation of $E_{\epsilon}$.

Numerically, the Laplacian term $\Delta z^{n+1}$ is computed by the standard 5-pixel stencil, i.e., with $h$ denoting the grid size,

$$
h^{2} \Delta z=z_{i-1, j}+z_{i, j-1}+z_{i+1, j}+z_{i, j+1}-4 z_{i, j} .
$$

This could further lead to the Jacobi type iteration when the central pixel $z_{i, j}$ is assigned to the time step $n+1$ while the other four neighbors still stay at the step $n$.

We now elaborate on how to develop proper linearization schemes for the nonlinear terms that involve sine and sinc. For the second term with $\sin 2 \pi z_{i, j}^{n+1}$, we use $\frac{\sin 2 \pi z_{i, j}^{n}}{z^{n}} z_{i, j}^{n+1}=2 \pi z_{i, j}^{n+1} \operatorname{sinc}\left(2 z_{i, j}^{n}\right)$ for linearization. This is inspired by the closely related problem of finding a solution to the nonlinear equation $\sin x=a$ for a given $a \in(0,1)$ and on $[0, \pi / 2]$. An effective iteration scheme is given by the same linearization technique:

$$
\frac{\sin x^{n}}{x^{n}} x^{n+1}=a, \quad \text { or equivalently, } \quad x^{n+1}=a \frac{x^{n}}{\sin x^{n}}=\Phi\left(x^{n}\right),
$$


where $\Phi(x)=\frac{a x}{\sin x}$. A remarkable property is that for $x \in[0, \pi / 2]$ and any given $a \in(0,1), \Phi$ is a contractive mapping, i.e., $\max _{x \in[0, \pi / 2]}\left|\Phi^{\prime}(x)\right| \leq a<1$. In particular, the linearization iteration indeed leads to a unique fixed point $x^{*}, \frac{a x^{*}}{\sin x^{*}}=x^{*}$ by Picard's fixed point theorem. In addition, to avoid singularities in the denominators, small value $\delta, \delta \ll 1$, (e.g., $\delta=10^{-16}$, the MATLAB constant), is often added for numerical robustness.

For the fourth term on the left that involves the derivative of the sinc function, we similarly linearize it to

$$
\frac{d}{d z} \operatorname{sinc}^{2}\left(z_{i, j}^{n+1}-k\right)=2 \operatorname{sinc}\left(z_{i, j}^{n}-k\right)\left[\frac{\pi z \cos \pi z-\sin \pi z}{\pi z^{3}}\right]_{z=z_{i, j}^{n}-k}\left(z_{i, j}^{n+1}-k\right) .
$$

Combining all the above steps of finite-difference discretization and function linearization, in the case when the Jacobi iteration is adopted for the Laplacian, we attain the following iteration scheme: at each step $n$,

$$
\begin{aligned}
& \left\{8 \epsilon+\frac{\pi}{\epsilon}\left(\frac{\sin 2 \pi z_{i, j}^{n}}{z_{i, j}^{n}}+2 \pi\right)\right. \\
& \left.+\lambda \sum_{k=0}^{K-1}\left|u_{i, j}-C_{k}\right|^{2}\left(\frac{2 \pi^{2}}{3}+2 \operatorname{sinc}\left(z_{i, j}^{n}-k\right) \frac{\frac{d}{d z} \operatorname{sinc}\left(z_{i, j}^{n}-k\right)}{z_{i, j}^{n}-k}\right)\right\} z_{i, j}^{n+1} \\
= & 2 \epsilon\left(z_{i-1, j}^{n}+z_{i, j-1}^{n}+z_{i+1, j}^{n}+z_{i, j+1}^{n}\right)+\frac{2 \pi^{2}}{\epsilon} z_{i, j}^{n} \\
& +\lambda \sum_{k=0}^{K-1}\left|u_{i, j}-C_{k}\right|^{2}\left(\frac{2 \pi^{2}}{3} z_{i, j}^{n}+2 k \operatorname{sinc}\left(z_{i, j}^{n}-k\right) \frac{\frac{d}{d z} \operatorname{sinc}\left(z_{i, j}^{n}-k\right)}{z_{i, j}^{n}-k}\right) .
\end{aligned}
$$

The Neumann natural boundary condition is imposed along the boundary of the image domain. The detailed numerical analysis on the CCCP method augmented with all the above linearization techniques is interesting but foreseeably involved. This offers an intriguing open problem to the numerical analysis community.

Finally, once the phase field $z$ is solved from the system, in order to extract each phase or segment, we apply the hard thresholding decision rule: $k-\frac{1}{2} \leq z<k+\frac{1}{2}$ for each individual $k$-th phase. A simple morphological transformation (opening) is also employed to remove any spurious dots due to the hard thresholding. Instead of hard thresholding, it is also possible to adopt a slightly more complex local decision rule based on windowing, which will then make the morphological operation obsolete.

\subsection{Numerical experiments}

In this subsection, we present some generic experimental results based on the theories and computational schemes developed above. In all the experiments, a given image is always normalized to the canonical gray interval $[0,1]$, and the bandwidth parameter (or the diffuse scale) $\epsilon$ is in the order of a few pixels. Furthermore, inspired by the simulated annealing technique in stochastic image processing (e.g., Geman and Geman [23]) and Gibbs' random fields, we have also experimented with dynamically decreasing $\epsilon$ 's to speed up convergence, for example, adopting $\epsilon_{1}$ in the first 50 iterations, while $\epsilon_{2}=\epsilon_{1} / 2$ for the rest.

Regarding the initial guess for the phase field $z$, we have typically adopted random values between -0.5 to $K+0.5$ as mentioned in the theory (the set $\mathcal{A}_{K}$ ). For complex images with large variances in homogeneous regions or with many phases, weak supervision can be used for the initial values, i.e., initial $C_{k}$ values can be estimated from the assigned supervised "seed" regions. For more discussion on weak supervision and automated stochastic supervision (based on patch statistics), we refer to the recent works of Shen [46], and $\mathrm{Li}$ and Perona $[27,26]$.

The first two experiments are on two generic synthetic images. In Figure 1, it is shown that the proposed model works well with a noisy image containing a generic T-junction, a universal singular structure crucial in visual perception (e.g., Nitzberg, et al. [37]). 


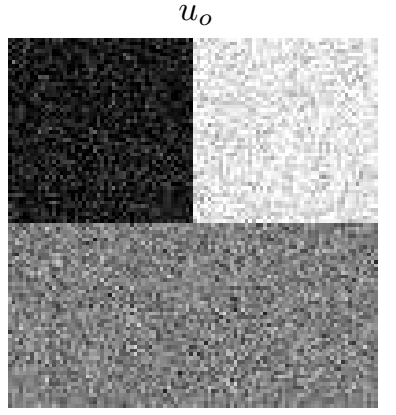

$z=0$

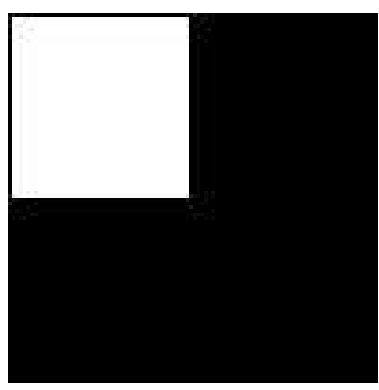

$z$

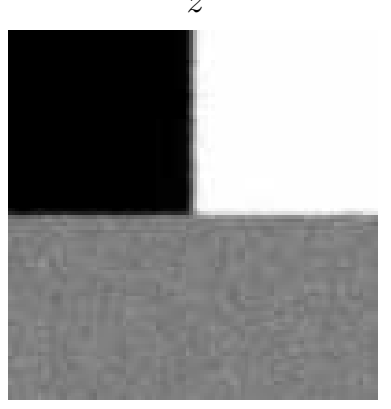

$z=1$

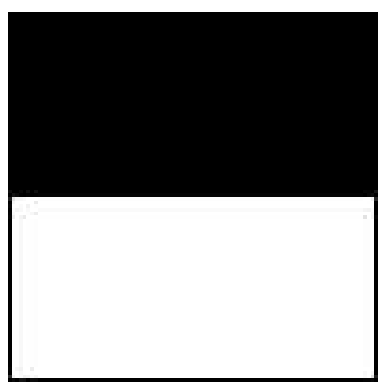

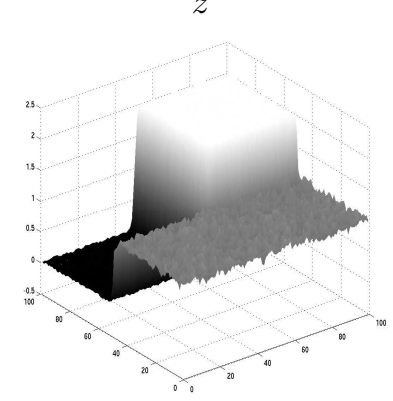

$z=2$

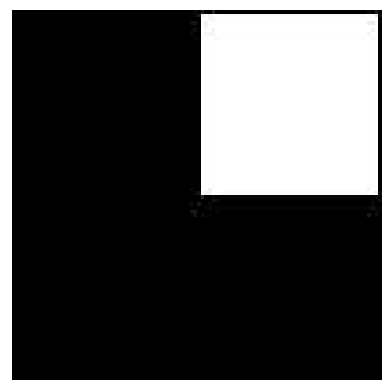

Figure 1: A noisy synthetic image $u_{o}$ containing a generic T-junction (upper left), the phase field $z$ computed by the proposed sine-sinc model (upper middle and right), and the three individual phases (the bottom row). The example shows that the model is robust to noise and reconstructs well the geometry.

Figure 2 shows another more complex synthetic image that contains several generic visual structures, including an internal hole, occlusion and stacked objects, T-junctions, singular junctions where multiple objects (or phases) meet, as well as thin passages that reveal the bottleneck effect (see the recent work of Kohn and Slastikov in material sciences [25] for asymptotic bottleneck analysis in phase transitions). In this case, with $K=5$ phases, initial condition is weakly supervised. When this is experimented with random $K$ values, it results in phase merging or empty phases.

The next three experiments demonstrate the performance of the proposed model and its computational schemes on three generic natural images.

Figure 3 shows the application of the proposed model to an MRI brain image. Even though the intensities fluctuate severely and the boundaries are complex, the proposed method has done a satisfactory job in separating the major different phases. Shown on top of the original image are the three small patches that are in practice easily supervised by a radiologist.

The final two examples in Figure 4 and 5 both involve color images for which the RGB color space has been employed. We have adopted the Euclidean metric of three color channels as in [50]:

$$
\left|u_{o}^{R}-C_{k}^{R}\right|^{2}+\left|u_{o}^{G}-C_{k}^{G}\right|^{2}+\left|u_{o}^{B}-C_{k}^{B}\right|^{2}
$$

where $u_{o}^{R}, u_{o}^{G}, u_{o}^{B}$ correspond to the red, green, blue channels of the given color image $u_{o}$. ( This may not be optimal for color perception, e.g., $[10,11])$. These two examples further demonstrate the flexibility of the proposed model and its computational algorithm.

\section{Conclusion}

In this paper, we propose a new multiphase segmentation model based on the celebrated phase transition model of Modica and Mortola [34] in material sciences, fluid mechanics, and the $\Gamma$-convergence theory. The 


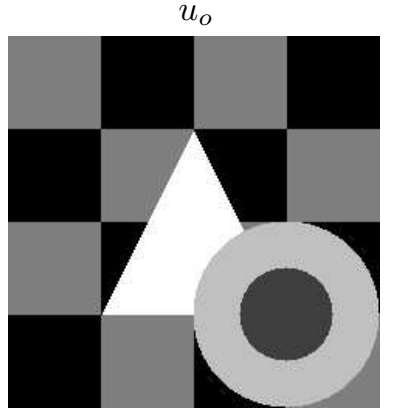

$z=0$

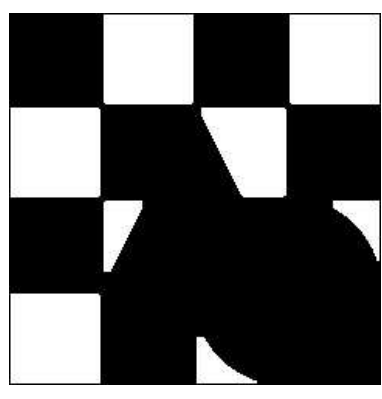

$z=2$

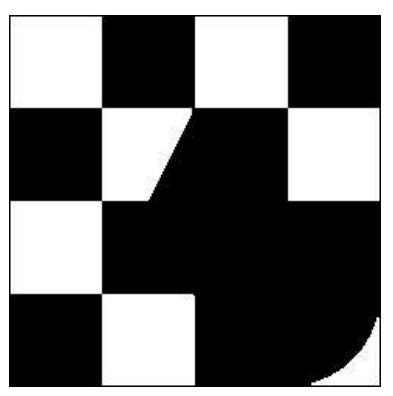

$z$

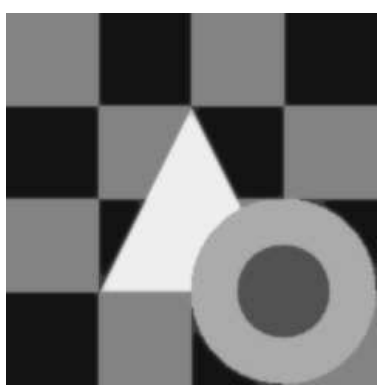

$z=1$

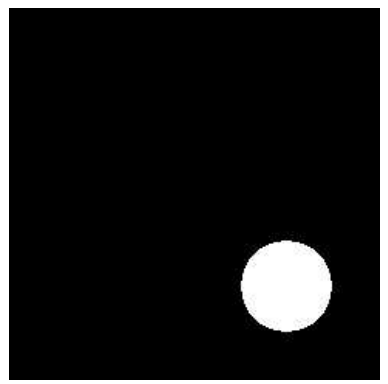

$z=3$

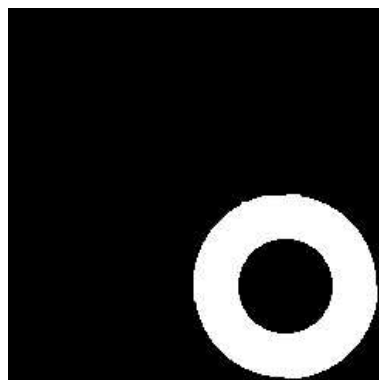

$z$

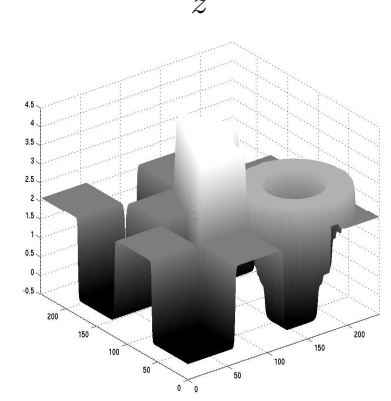

$z=4$

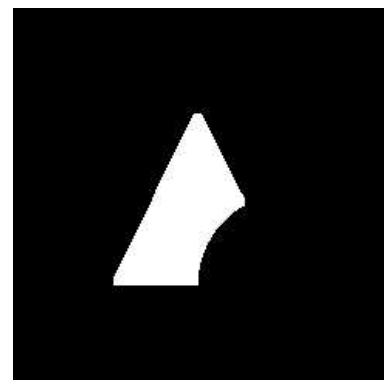

Figure 2: A complex synthetic image $u_{o}$ with multiple objects and several generic visual structures. Image size is $240 \times 240, \epsilon=2$ (pixels), and $\lambda=15$ (scaled by the grid size). The calculated $C_{k}$ values are $0,0.16$, $0.50,0.74,0.97$, respectively.

sine-sinc model properly synchronizes the fitting term for the given image with the regularity term for the diffuse interfaces. Mathematical analysis has been developed for the $\Gamma$-convergence behavior of the model and the existence of its minimizers. We have also developed in detail the convex-splitting or the CCCP algorithm for minimizing the non-convex energy functional. Several numerical experiments on both generic synthetic and natural images have demonstrated the satisfying performance of the proposed model and its algorithm.

It is our belief that the interplay and integration between physics and information technologies will further blossom in the near future. The current work is a typical example that has substantially benefited from numerous existing contributions in these two fields.

\section{Acknowledgments}

Jung is grateful to Prof. Jin Keun Seo for his support and encouragement. Kang would like to thank IMA for supporting a long term visit to IMA during the thematic year on Imaging which has made this collaboration 


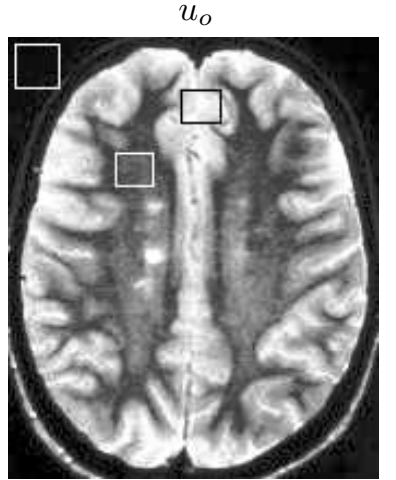

$z=0$

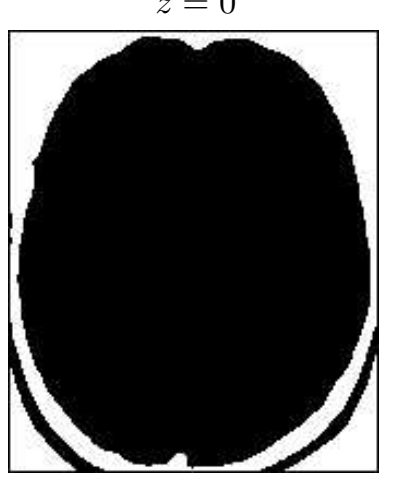

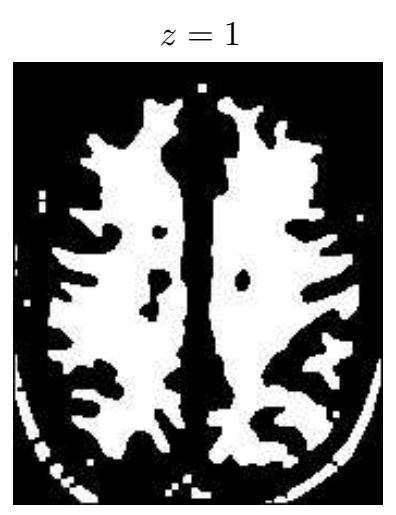

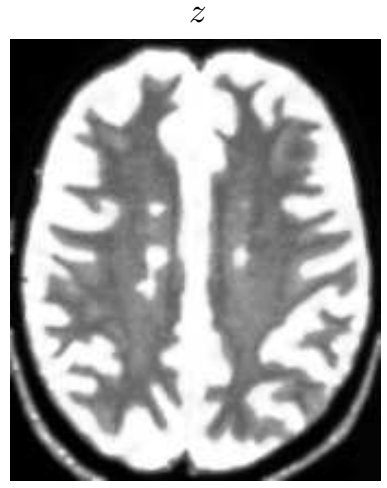

$z=2$

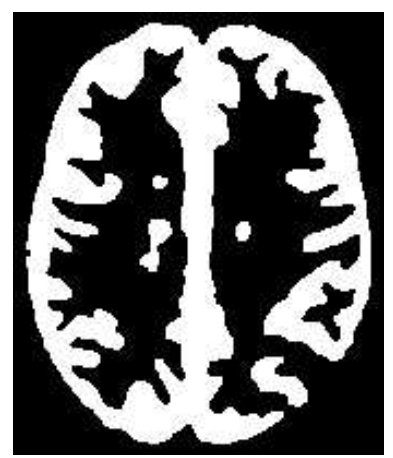

Figure 3: The performance of the proposed Modica-Mortola sine-sinc model to the segmentation of a MRI brain image. Shown on top of the original image (upper left) are the three "seed" phase patches which are often easily supervised by a radiologist. The resolution details in the segmented phases depend upon the bandwidth parameter $\epsilon$ ( $\epsilon=2$ (pixels) for this particular output).

possible. Shen would like to thank his dear teacher and friend Riccardo March for kindly educating him the $\Gamma$-convergence theory with fun and joy through all these years, and also dear friends Alan Yuille and Andrea Bertozzi for the enlightenment on the convex splitting or the CCCP algorithm.

\section{References}

[1] G. Alberti. Variational models for phase transitions, an approach via $\Gamma$-convergence. In Calculus of variations and partial differential equations (Pisa, 1996), pages 95-114. Springer, Berlin, 2000.

[2] L. Ambrosio and V. M. Tortorelli. Approximation of functionals depending on jumps by elliptic functionals via $\Gamma$-convergence. Comm. Pure Appl. Math., 43:999-1036, 1990.

[3] L. Ambrosio and V. M. Tortorelli. On the approximation of free discontinuity problems. Boll. Un. Mat. Ital., 6-B:105-123, 1992.

[4] A. Bertozzi, S. Esedoglu, and A. Gilette. Inpainting of binary images using the Cahn-Hilliard equation. UCLA CAM Rep.06-0\%, 2006.

[5] B. Bourdin and A. Chambolle. Implementation of an adaptive finite-element approximation of the Mumford-Shah functional. Numer. Math., 85(4):609-646, 2000. 


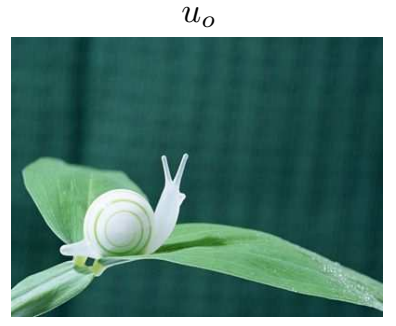

$z=0$

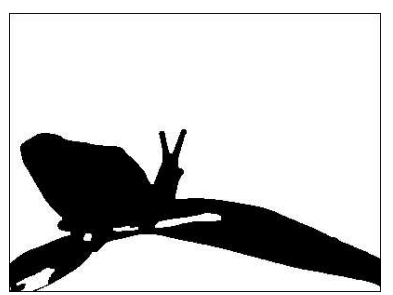

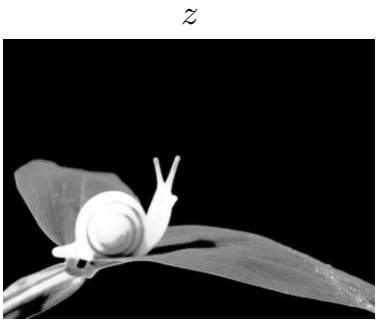

$z=2$

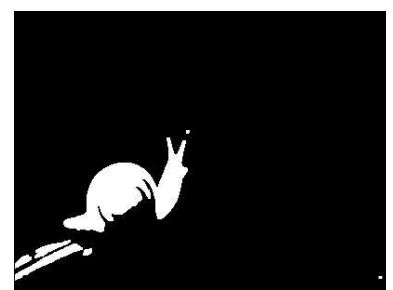

Figure 4: Segmenting a color image with a milky snail on green leaves by the proposed Modica-Mortola sine-sinc model. The RGB channels enter the model by a simple application of the Euclidean distance.

[6] A. Braides. $\Gamma$-convergence for beginners, volume 22 of Oxford Lecture Series in Mathematics and its Applications. Oxford University Press, Oxford, 2002.

[7] J.W. Cahn and J.E. Hilliard. Free energy of a non-uniform system I - Interfacial free energy. J. Chem. Phys., 28:258-267, 1958.

[8] A. Chambolle. Image segmentation by variational methods: Mumford and Shah functional and the discrete approximations. SIAM J. Appl. Math., 55(3):827-863, 1995.

[9] A. Chambolle. Finite-differences discretizations of the Mumford-Shah functional. M2AN Math. Model. Numer. Anal., 33(2):261-288, 1999.

[10] T. F. Chan, S.-H. Kang, and J. Shen. Total variation denoising and enhancement of color images based on the CB and HSV color models. J. Visual Comm. Image Rep., 12(4):422-435, 2001.

[11] T. F. Chan and J. Shen. Variational restoration of non-flat image features: models and algorithms. SIAM J. Appl. Math., 61(4):1338-1361, 2000.

[12] T. F. Chan, J. Shen, and L. Vese. Variational PDE models in image processing. Amer. Math. Soc. Notice, 50:14-26, 2003.

[13] T. F. Chan and L. Vese. A level set algorithm for minimizing the Mumford-Shah functional in image processing. IEEE/Computer Society Proceedings of the 1st IEEE Workshop on "Variational and Level Set Methods in Computer Vision", pages 161-168, 2001.

[14] T.F. Chan and J. Shen. Image processing and analysis: variational, PDE, wavelet, and stochastic methods. Society for Industrial and Applied Mathematics (SIAM), Philadelphia, PA, 2005.

[15] T.F. Chan and L.A. Vese. Active contours without edges. IEEE Trans. on Image Process., 10(2):266$277,2001$.

[16] T.F. Chan and L.A. Vese. A multiphase level set framework for image segmentation using the Mumford and Shah model. Int. J. Comp. Vision, 50(3):271-293, 2002. 

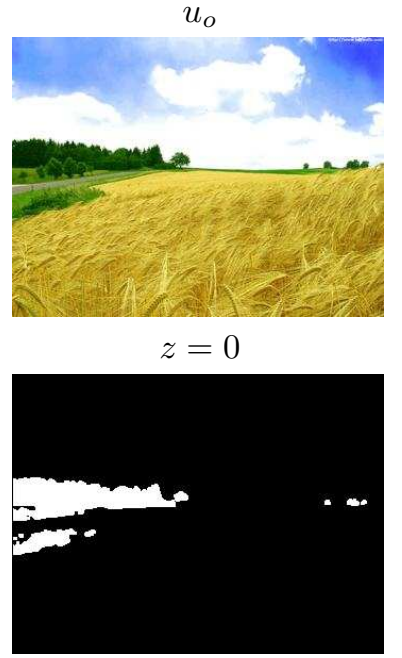

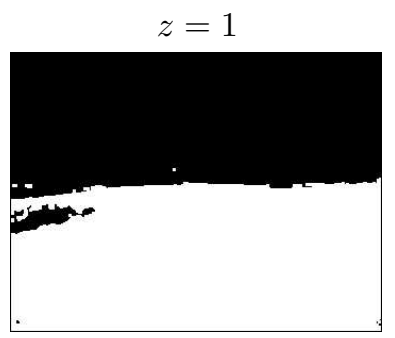

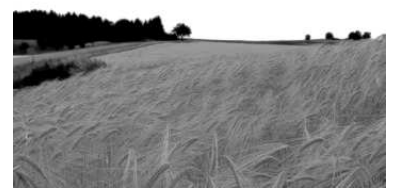

$z=2$

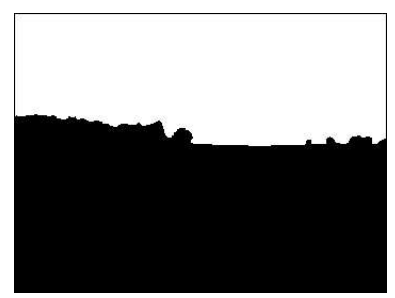

Figure 5: The proposed Modica-Mortola sine-sinc model performs satisfactorily on this outdoor color scene with a golden straw field, a greenish wood, and a sky.

[17] J. T. Chung and L. A. Vese. Image segmentation using a multilayer level-set approach. UCLA CAM Report 03-53, 2003.

[18] F. Cucker and S. Smale. On the mathematical foundations of learning. Bull. AMS, 39(1):1-49, 2001.

[19] I. Daubechies. Ten lectures on wavelets. SIAM, Philadelphia, 1992.

[20] S. Esedoglu and R. March. Segmentation with depth but without detecting junctions. J. Math. Imaging and Vision, 18, 2003.

[21] S. Esedoglu and J. Shen. Digital inpainting based on the Mumford-Shah-Euler image model. European J. Appl. Math., 13:353-370, 2002.

[22] D. Eyre. An unconditionally stable one-step scheme for gradient systems. Unpublished paper, 1998. www.math.utah.edu/ eyre/research/methods/stable.ps.

[23] S. Geman and D. Geman. Stochastic relaxation, Gibbs distributions, and the Bayesian restoration of images. IEEE Trans. Pattern Anal. Machine Intell., 6:721-741, 1984.

[24] E. De Giorgi and T. Franzoni. Su un tipo di convergenza variazionale. Atti Accad. Naz. Lincei Rend. Cl. Sci. Fis. Mat. Natur. (8), 58(6):842-850, 1975.

[25] R. V. Kohn and V. V. Slastikov. Geometrically constrained walls. Preprint, 2004.

[26] F.-F. Li and P. Perona. A Bayesian hierarchical model for learning natural scene categories. IEEE CVPR, 2(2):524-531, 2005.

[27] F.-F. Li, R. VanRullen, C. Koch, and P. Perona. Rapid natural scene categorization in the near absence of attention. Proc. Nat'l Acad. Sci. (USA), 99(14):9596-9601, 2002.

[28] J. Lie, M. Lysaker, and X.-C. Tai. A variant of the level set method and applications to image segmentation. Math. Comp., 75:1155-1174, 2006.

[29] R. March. Visual reconstruction with discontinuities using variational methods. Image Vision Comput., 10:30-38, 1992. 
[30] R. March and M. Dozio. A variational method for the recovery of smooth boundaries. Image Vision Comput., 15:705-712, 1997.

[31] G. Dal Maso. An introduction to $\Gamma$-convergence. Progress in Nonlinear Differential Equations and their Applications, 8. Birkhäuser Boston Inc., Boston, MA, 1993.

[32] Y. Meyer. Oscillating Patterns in Image Processing and Nonlinear Evolution Equations, volume 22 of University Lecture Series. AMS, Providence, 2001.

[33] L. Modica. The gradient theory of phase transitions and the minimal interface criterion. Arch. Rational Mech. Anal., 98(2):123-142, 1987.

[34] L. Modica and S. Mortola. Un esempio di $\Gamma^{-}$-convergenza. Boll. Un. Mat. Ital. B (5), 14(1):285-299, 1977.

[35] J.-M. Morel and S. Solimini. Variational Methods in Image Segmentation, volume 14 of Progress in Nonlinear Differential Equations and Their Applications. Birkhäuser, Boston, 1995.

[36] D. Mumford and J. Shah. Optimal approximations by piecewise smooth functions and associated variational problems. Comm. Pure Appl. Math., 42(5):577-685, 1989.

[37] M. Nitzberg, D. Mumford, and T. Shiota. Filtering, Segmentation, and Depth. Lecture Notes in Comp. Sci., Vol. 662. Springer-Verlag, Berlin, 1993.

[38] S. Osher and R. Fedkiw. Level set methods and dynamic implicit surfaces, volume 153 of Applied Mathematical Sciences. Springer-Verlag, New York, 2003.

[39] S. Osher and J. A. Sethian. Fronts propagating with curvature-dependent speed: Algorithms based on Hamilton-Jacobi formulations. J. Comput. Phys., 79(12), 1988.

[40] S. Osher, A. Solé, and L. Vese. Image decomposition and restoration using total variation minimization and the $H^{-1}$ norm. Multiscale Model. Simul., 1(3):349-370 (electronic), 2003.

[41] T. Poggio and S. Smale. The mathematics of learning: Dealing with data. Amer. Math. Soc. Notice, 50(5):537-544, 2003.

[42] B. Sandberg, T. Chan, and L. Vese. A level-set and gabor-based active contour algorithm for segmenting textured images. UCLA CAM report 02-39, 2002.

[43] J. A. Sethian. Level set methods and fast marching methods, volume 3 of Cambridge Monographs on Applied and Computational Mathematics. Cambridge University Press, Cambridge, second edition, 1999.

[44] J. Shen. Bayesian video dejittering by BV image model. SIAM J. Appl. Math., 64(5):1691-1708, 2004.

[45] J. Shen. Piecewise $H^{-1}+H^{0}+H^{1}$ images and the Mumford-Shah-Sobolev model for segmented image decomposition. AMRX Appl. Math. Res. Express, 4:143-167, 2005.

[46] J. Shen. A stochastic-variational model for soft mumford-shah segmentation. International Journal of Biomedical Imaging, Vol. 2006, ID92329, 2006.

[47] J. Shi and J. Malik. Normalized cuts and image segmentation. IEEE Trans. Pattern Anal. Machine Intell., 22(8):888-905, 2000.

[48] S. Smale and D.-X. Zhou. Shannon sampling and function reconstruction from point values. Bull. Amer. Math. Soc., 41:279-305, 2004.

[49] X.-C. Tai and T.F. Chan. A survey on multiple level set methods with applications for identifying piecewise constant functions. International J. Numer. Anal. Modelling, 1(1):25-48, 2004. 
[50] L.A. Vese T.F. Chan, B.Y. Sandberg. Active contours without edges for vector-valued images. J. Visual Comm. Image Rep., 11(2):130-141, 2000.

[51] Z.W. Tu and S.C. Zhu. Image segmentation by Data-Driven Markov Chain Monte Carlo. IEEE Trans. Pattern Anal. Machine Intell., 24(5):657-673, 2002.

[52] C. Vogel. Computational Methods for Inverse Problems. SIAM, Philadelphia, 2002.

[53] B.P. Vollmayr-Lee and A.D. Rutenberg. Fast and accurate coarsening simulation with an unconditionally stable time step. Physical Review E, 68(6):066703, 2003.

[54] A.L. Yuille and A. Rangarajan. The concave-convex procedure (CCCP). Neural Computation, 15(4):915-936, 2003. 